\title{
Analisi quantitativa della Risonanza Magnetica Nucleare T2-pesata come potenziale indicatore di risposta agli analoghi della somatostatina nell'acromegalia di nuova diagnosi
}

\author{
Salvatore Cannavò ${ }^{1}$
}

Pubblicato online: 28 marzo 2017

C Springer International Publishing AG 2017

\section{Commento a:}

Quantitative analyses of T2-weighted MRI as a potential marker for response to somatostatin analogs in newly diagnosed acromegaly.

A. Heck, G. Ringstad, K.E. Emblem, O. Casar-Borota, J. Bollerslev.

Endocrine (2016) 52:333-343

Gli analoghi della somatostatina ( $\mathrm{SSa}$ ) rappresentano il trattamento medico di prima linea per l'acromegalia, dopo l'insuccesso chirurgico. In molti casi essi sono utilizzati anche prima della chirurgia, per indurre l'attenuazione di complicanze sistemiche che aumentano il rischio anestesiologico, o come terapia di scelta in pazienti non candidabili all'intervento o con una ridotta probabilità di successo chirurgico. Infatti, l'indicazione elettiva all'utilizzo di tali farmaci si rafforza progressivamente al crescere delle dimensioni dell'adenoma ipofisario. Tuttavia, la sensibilità agli SSa è variabile da caso a caso e un adeguato controllo dei livelli sia di GH che di IGF-1 si verifica in meno del 35-40\% dei casi. Pertanto, l'individuazione di parametri predittivi di risposta al trattamento prolungato, siano essi clinici, biochimici, medico-nucleari o morfologici, rappresenta un argomento ampiamente dibattuto da quando questa classe di farmaci è stata introdotta nella pratica clinica.

Alcuni studi hanno dimostrato che gli adenomi ipofisari GH-secernenti densamente granulati sono più frequentemente sensibili agli SSa e che essi appaiono ipointen- si nelle sequenze T2-pesate, quando studiati con la risonanza magnetica nucleare (RMN). Tuttavia, il criterio di valutazione comunemente utilizzato è empirico e soggettivo, essendo basato sul giudizio prettamente qualitativo del neuroradiologo.

Lo studio di Heck et al. ha proposto un approccio quantitativo basato sulla misurazione oggettiva dell'intensità, omogeneità e dispersione del segnale nelle sequenze T2pesate della RMN 1.5 Tesla senza mezzo di contrasto, mediante un software dedicato. Per la validazione di tale metodologia, gli autori hanno rivalutato retrospettivamente 34 pazienti acromegalici trattati con SSa per una mediana di 6 mesi, dopo essere stati sottoposti a RMN della regione ipotalamo-ipofisaria. Lo studio ha confermato che l'analisi quantitativa dell'intensità e della dispersione dimostra un'elevata accuratezza per la potenziale identificazione dei pazienti responsivi agli $\mathrm{SSa}$, impiegati come terapia di prima linea. Tuttavia, la classificazione convenzionale in adenomi ipointensi, isointensi e iperintensi, basata su criteri valutativi visivi, fornisce informazioni altrettanto accurate e può essere proposta come metodo validato per la pratica clinica, riservando l'uso del metodo quantitativo a finalità di ricerca clinica e traslazionale.

In conclusione, la valutazione dell'intensità del segnale nelle sequenze RMN T2-pesate consente l'identificazione dei pazienti con tumori GH-secernenti scarsamente granulati che sono generalmente più resistenti al trattamento con gli SSa di prima generazione (octreotide e lanreotide).
$凶$ S. Cannavò

cannavos@unime.it

1 Dipartimento di Medicina Clinica e Sperimentale, Università di Messina, Messina, Italia 